Transportation Research Forum

The Impacts of Emergency Vehicle Signal Preemption on Urban Traffic Speed

Author(s): Hualiang (Harry) Teng, Valerian Kwigizile, Gang Xie, Mohamed Kaseko, and A. Reed Gibby

Source: Journal of the Transportation Research Forum, Vol. 49, No. 1 (Spring 2010), pp. 69-79

Published by: Transportation Research Forum

Stable URL: http://www.trforum.org/journal

The Transportation Research Forum, founded in 1958, is an independent, nonprofit organization of transportation professionals who conduct, use, and benefit from research. Its purpose is to provide an impartial meeting ground for carriers, shippers, government officials, consultants, university researchers, suppliers, and others seeking exchange of information and ideas related to both passenger and freight transportation. More information on the Transportation Research Forum can be found on the Web at www.trforum.org. 


\title{
The Impacts of Emergency Vehicle Signal Preemption on Urban Traffic Speed
}

\author{
by Hualiang (Harry) Teng, Valerian Kwigizile, Gang Xie, Mohamed Kaseko, \\ and A. Reed Gibby
}

We used GPS data from paratransit vehicles to evaluate the impact of emergency vehicles on urban traffic speeds. The results indicate that speed variance is significantly higher during emergency preemption and the mean speeds of traffic flowing in the same direction as the emergency vehicle and on crossing streets are lower during preemption than during normal conditions. Regression results indicate that traffic on major arterials and traffic in the opposite direction of the emergency vehicle tend to have higher speed during signal preemption. Signal preemption during peak periods and duration of preemption had a significant negative impact on traffic speeds. Also, the transition time has a negative impact on traffic speeds. The authors recommend further research on how to optimize (minimize) the preemption duration as well as transition time. Also, the impact of median type and number of lanes should be evaluated.

\section{INTRODUCTION}

Emergency vehicle signal preemption interrupts regular traffic signal operations to allow emergency vehicles to pass through intersections safely with minimum delay. Usually, a "signal" from an emergency vehicle is sent to a detector device that notifies the traffic signal controller so that signal display and timing are altered in favor of the emergency vehicle. When the signal display facing the emergency vehicle is red, to satisfy safety requirements for vehicles and pedestrians, the green signal display for the crossing street traffic will continue long enough to satisfy the timing needs for the cross street vehicles or pedestrians. Then the traffic signal changes so the emergency vehicle receives a green indication before entering the intersection. If the signal facing the emergency vehicle is green, it will be extended until the emergency vehicle enters the intersection. After the passage of the emergency vehicle, the traffic signal controller returns to normal operation. If the traffic signal is coordinated with other signals, it will operate through a transition, when signal phasing and timing transition to normal operation. After this transition period, the traffic signals function according to the normal traffic signal coordination and timing plan.

It has been observed that emergency vehicle drivers adapt to various traffic operating conditions differently. Similarly, drivers in general alter their driving behavior correspondingly. During peak periods, emergency vehicles sometimes are caught in the heavy traffic congestion and cannot reach a downstream intersection (next intersection in the direction of the emergency vehicle) with the green time provided. In this situation, emergency vehicles will most likely need to cross the median and enter an opposing lane of traffic in order to bypass the congested roadway segment. During the off-peak period, when traffic is lighter, the traffic approaching the downstream intersection usually maneuvers out of the path of emergency vehicles. Some vehicles may trail the emergency vehicle and pass through the downstream intersection at high speeds. The vehicles moving in the opposite direction yielding to an emergency vehicle often miss the green phase, experiencing additional delay. Those vehicles moving in the same direction as the emergency vehicle and yielding to it while approaching the preempted traffic signal also experience delay. The traffic on the cross street will suffer a reduced green time or a longer red time. However, the total daily approach delay will generally not be significant unless the number of preemptions becomes excessive.

A significant amount of time may be required for traffic signals to transition back to normal operations after a preemption. If there are numerous preemptions per day at an intersection, the 
opportunity for normal operation could be small. For example, in the Las Vegas Urbanized Area, it was reported that some traffic signals had as many as 400 emergency preemptions in a week (Kaseko and Teng 2005). Considering the additional time for the transition needed for traffic signals recovering back to coordination from preemption, traffic signals at these intersections may have a small chance to operate regularly. Thus, there is a need to investigate the conditions under which preemptions caused significant traffic delay. The results from such studies can be used by traffic engineers for establishing signal preemption strategies.

Several studies have been conducted on the impact of traffic signal preemption for emergency vehicles. Different forms of traffic simulation models (microscopic, which simulates single vehicledriver unit dynamics; or macroscopic, which simulates a group of vehicles) were employed in these studies. To some extent, the simulation models used in these studies may not fully model the driving behavior of emergency vehicle operators, such as using the travel lanes of opposite direction. They may not model the reaction of the general traffic for giving the right of way to emergency vehicles. Also, there have been conflicting findings reported from studies employing simulation. The methodology employed in this study uses "real" data that represent driver behavior and reactions to the presence of emergency vehicles.

The study presented in this paper evaluates the impact of signal preemptions on traffic operations. Instead of using simulation, it utilizes the data from a global positioning system (GPS) installed on para-transit vehicles that are operated by the Regional Transportation Commission in the Las Vegas area of Southern Nevada. Typically, these vehicles are vans and travel to locations in the valley to provide service to eligible customers. Advanced reservations are required. Paratransit vehicles involve fewer stops than conventional transit buses and were therefore assumed to have travel patterns and operating characteristics similar to regular traffic, particularly from the perspective of responding to emergency vehicles. In this study, the GPS data were processed to derive speed and travel time data for the roadway segments where the para-transit vehicles traveled. Also, the signal event logs for intersections where the para-transit vehicles traversed were used. It should be noted that both GPS data and signal event logs contain time information. Using this time information, the speed and travel time data were then identified as to whether or not they were associated with signal preemptions. Based on the traffic speed data extracted for the normal and preemption conditions, tests of hypotheses were performed to compare the means and variances of the traffic speeds during preemption and normal operations. The results from testing whether the variance of traffic speed during preemption is greater than that during normal operations can determine whether or not emergency preemptions caused turbulences in traffic flow that may have some implications for safety. The test of whether the mean of traffic speeds changes significantly after preemption provides an evaluation of its overall impact on traffic flow. Regression analysis was also performed in this study to identify the factors associated with turbulences in traffic speeds during preemption operations.

This paper contains six sections. The first section provided an introduction to the problems to be addressed in this study and described the methodology. In the second section, a literature review on previous studies related to the evaluation of the impact of emergency preemption on traffic operations is provided. The third section presents the methodology used to evaluate the impact of preemptions on traffic operations. In the fourth section, the data collection is described, including the data sources, the assumptions used in extracting data and the validation of the data. The fifth section presents the analysis, and the last section provides conclusions, recommendations and future study needs.

\section{LITERATURE REVIEW}

The concerns on the impacts of emergency preemptions on the mobility of general traffic are reflected in a survey conducted by Gifford et al. (2001). The authors stressed that although emergency and transit agency personnel are interested in signal preemption technology, they and 
the other stakeholders have significant questions and reservations to be considered in the adoption and deployment of preemption and priority systems. Some studies, such as Nelson and Bullock (2000), Bullock et al. (1999), and Yun et al. (2007), evaluated the impact of emergency preemptions using the "hardware-in-the-loop" (HITL) simulation technique. In the HITL technique, realistic simulations of emergency preemptions are performed in a real time virtual environment-it combines a microscopic simulation program and an actual traffic controller. One of the studies concluded that even though HITL has the capability to evaluate the preemption operations, the huge computational time requirements may be a significant disadvantage of the technique. Casturi et al. (2000) developed an advanced macroscopic model to represent emergency vehicles by which the impacts of emergency vehicles on general traffic were evaluated.

Nelson and Bullock (2000) investigated the impact of emergency vehicle signal preemption on closely spaced arterial traffic signals. It simulated a network consisting of four coordinated intersections on a principal arterial, seven emergency vehicle paths and three different transition algorithms. The designed simulation scenarios considered different time periods, each of which was associated with a different traffic volume and numbers of preemptions. The results showed that the impact of single preemption on the overall travel time and delay for the modeled network was minimal. For most scenarios, the impact of preemption was the least for the arterial and crossing streets when implementing the smooth transition strategy. The impact of preemption was more severe when multiple preemptions happened over a short time period. The following key factors were found to affect the impact of emergency vehicle signal preemption: (1) distance between intersections; (2) transition algorithms; (3) saturation of intersection; (4) duration of preemption; and (5) amount of slack time (the difference between the amount of time emergency preemption takes and the amount of time allocated in each cycle) available in each signal cycle. Similar findings can be found in the study by Bullock et al. (1999).

Yun et al. (2007) evaluated various preemption strategies for the case where an emergency vehicle arrives at a single approach on a coordinated-actuated traffic signal system. A coordinatedactuated traffic signal system is a series of coordinated signals that use information on current traffic demand (as received from sensors, etc.) to alter one or more aspects of the signal timing. The roadway section simulated in this study was an urban corridor that included four coordinatedactuated signals. Different scenarios consisting of a different number of cycles and sequences of phases for crossing streets were evaluated. They found that even a single preemption call could cause significant increases in delays and travel times.

Casturi et al. (2000) developed an advanced macroscopic model to assess the impact of emergency signal preemption on traffic delay. To mimic the pullover effect of the general traffic in response to an emergency vehicle, a mechanism to represent a capacity reduction factor was developed. To test the model, a simple network of a one-lane, one-way street with three intersections was constructed, and five test scenarios consisting of different frequencies of emergency vehicles and headways were designed. The impact of signal preemption on the general traffic, especially on crossing streets, was evaluated.

\section{RESEARCH METHODOLOGY}

\section{Hypothesis Testing of Speeds in Preemptions}

To study whether signal preemptions have any impact on traffic conditions, an F-test was performed first on whether or not the variances of traffic speeds during preemption and normal operations were the same. A reason for this test is that it has been perceived that some vehicles may speed up by trailing emergency vehicles, and others may be slowed down, yielding their right of ways to emergency vehicles. Due to this perception, the variance of vehicle speeds may increase due to emergency vehicle preemption of traffic lights. The variance of the speed was tested first for three different locations of the general traffic relative to the path of an emergency vehicle: (1) in the same 
direction of emergency vehicles, (2) in the opposite direction of emergency vehicles, and (3) on crossing streets.

In performing the F-test, the null hypothesis is that there is no significant difference between the variance of the speed during the preemption $\left(\sigma_{P}^{2}\right)$ and normal operations $\left(\sigma_{N}^{2}\right)$. The alternative hypothesis is that the variance during preemption is greater than it is during normal operations. These two hypotheses can be written as:

(1) $H_{0}: \sigma_{N}^{2}=\sigma_{P}^{2}$;

(2) $H_{1}: \sigma_{N}^{2}<\sigma_{P}^{2}$

The F-test statistic can be derived as

(3) $F=\frac{\sigma_{N}^{2}}{\sigma_{P}^{2}}$

The significance level $\alpha$ is chosen to be 0.05 . The hypothesis of the two variances being equal is rejected if

(4) $\mathrm{F}<\mathrm{F}_{\left(1-\alpha, n_{1}-1, n_{2}-1\right)}$.

In this formula, $n_{1}$ and $n_{2}$ represent sample sizes when traffic is in preemption and normal conditions, respectively.

After the test on the variance of traffic speed, t-tests were performed to determine whether signal preemptions increase or decrease traffic speed, or whether it remains the same. The null hypothesis is that there is no difference between mean traffic speeds during preemption and normal operations, which can be written as

(5) $H_{0}: S^{P}=S^{N}$

where $S^{N}$ and $S^{P}$ represent the averages of the traffic speeds during the normal and preemption operations, respectively. The alternative hypothesis is preemptions speeding up traffic, which can be expressed as:

(6) $H_{1}: S^{P}>S^{N}$.

Another alternative hypothesis is for slowing down traffic, which is:

(7) $H_{1}: S^{P}<S^{N}$

The t-test statistic can be written as:

(8) $t=\frac{\left(S^{N}-S^{P}\right)}{\sqrt{\frac{\sigma_{N}^{2}}{n_{1}}+\frac{\sigma_{P}^{2}}{n_{2}}}}$. 
The significance level $\alpha$ is chosen to be 0.05 . The conditions for accepting these two alternative hypotheses expressed in Equations (6) and (7) can be written as:

(9) $t \geq t_{\alpha}(m)$ for an upper one tailed test;

(10) $t \leq-t_{\alpha}(m)$ for a lower one tailed test;

where $m$ represents degree of freedom. If the variances were found to be equal, then:

(11) $m=n_{1}+n_{2}-2$.

\section{Regression Analysis of Traffic Speeds in Emergency Preemption}

To identify the factors that influence the speed of general traffic during preemption, a linear regression model of the following form was developed:

(12) $S_{j}^{P} \quad \beta^{\prime} x_{i j}+\varepsilon_{j}, j=1, \ldots, M$ and $i=1, \ldots, K$,

where $x_{i j}$ denotes the vector of factors $K$ that influence the traffic speed during preemption operations, and $\varepsilon_{j}$ is a random error. $M$ represents the number of observations.

\section{DATA COLLECTION AND PROCESSING}

In this study, speed and travel time data were collected through GPS devices installed on the paratransit vehicles in the Las Vegas area. Unlike the Citizens Area Transit (CAT), a fixed route system, para-transit in Las Vegas is a special bus system serving disabled or senior citizens. Para-transit service is provided 24 hours a day, seven days a week, and it operates not only within the urbanized area, but also outside the urbanized area as required. Due to their special services, para-transit vehicles have fewer stops than fixed-route bus services. From this perspective, para-transit vehicles can be used as surrogates of regular traffic from the operation perspective. There are about 150 paratransit vehicles in Las Vegas, each equipped with GPS devices to determine its location in real time.

The Regional Transportation Commission (RTC) of Southern Nevada operates the paratransit services and provided the GPS data for over 100 days for this study. These data include the identifications of the vehicles and their locations represented as longitudes and latitudes with associated time and date. Since these GPS data did not contain any information about road segments the vehicles were running on, they were processed to identify their locations. The longitude and latitude were overlaid on a map of Las Vegas to identify the location of the para-transit at a given time and date. The speeds of the para-transit vehicles were then derived by processing the GPS route data (longitudes, latitudes and time) sequentially in the order they were traversed. Most road segments were found to have more than one traffic speed data point. Based on these data, their mean and variance were calculated.

To validate the derived travel time and speed, we used the data from a travel time study conducted by a consulting firm (Kaseko and Teng 2005). In that study, several test cars were driven on the road network that covered a majority of the major arterials in the Las Vegas area. Travel time data were collected during peak and off-peak hours. Note that the travel times and speed data in the study conducted by the consulting firm were derived based on the road segments and corridors defined in a travel demand model that was maintained by the RTC, while the travel times and speeds derived in our emergency preemption study were based on actual road segments on a GIS map that were shorter than the road segments defined in the travel demand model. Therefore, the GIS segment-based travel speeds were aggregated for the segments and corridors that were defined in the travel demand model prior to comparing the speed data collected by the consulting firm and 
the data we derived from GPS data. Comparison results indicated that the speeds derived from the GPS data were lower than those collected by the consulting firm. There are several reasons for the discrepancies. One is that speeds in our study were based on para-transit vehicles, which were basically vans. This type of vehicle usually runs at a lower speed than automobiles, which were the test vehicles employed in the study by the consulting firm. It is assumed that this difference between the speeds measured by the consulting firm and those we derived from GPS data would not influence the results of the evaluation in our study. The reason for this assumption is the fact that the evaluation in this study was based on the comparison between traffic speeds during preemption and normal operations, both derived based on para-transit vehicles. Any bias in the traffic speeds derived based on para-transit vehicle would be eliminated in the comparison.

To identify the travel time/speeds under the influence of preemptions, the location and length of signal preemptions were identified based on the traffic signal data file and traffic signal event $\log$ data provided by the RTC. Thirty-seven (37) days (from January to June, 2006) of traffic signal event $\log$ data were processed and analyzed. Some of these data were incomplete (not for full 24 hours), and hence deleted. As a result, event logs for only 919 signals were obtained. Among these, 506 signals were found to have preemptions.

After identification of emergency preemptions, the traveling direction of the emergency vehicle was determined based on the information included in the traffic signal event logs and the traffic signal phase logs, identifying the traffic signals/intersections that were on the path followed by an emergency vehicle. These identified traffic signals were then used to derive the route that the emergency vehicle followed, using time sequence for start of preemptions at adjacent intersections. With the route derived for the emergency vehicle, its direction was finally determined. Then, the location of the para-transit vehicle relative to the emergency vehicle was correspondingly determined.

Determining whether or not a derived speed was influenced by an emergency preemption was based on whether or not the corresponding para-transit vehicle arrived at or departed from an intersection within a certain time period (one to six minutes) after the start of an emergency preemption at the intersection. The travel time/speed derived previously were labeled as being influenced if the calculated arrival or departure time for the para-transit vehicle is in this time range. The para-transit GPS data, traffic signal event log data, and traffic signal phase log data obtained for this study were for different days. It was found that they were commonly available for April 18 and 19, 2006, May 25 and 30, 2006, and June 1, 12, and 13, 2006. Thus, the samples of traffic speeds data from these seven days were processed to identify traffic speeds influenced by signal preemptions. To compare speeds influenced by signal preemptions to normal speed (speed when there is no preemptions), it was necessary to use the influenced speeds on those segments where normal speeds were available, too. Normal speeds were calculated by first matching the GPS data time with signal event log time. Then, the GPS data not coinciding with preemption were used to determine normal speed. A total of 978 segments met this condition and therefore generated a sample size of 978 influenced and normal speed data for analysis.

\section{DATA ANALYSIS}

\section{Hypothesis Testing of Vehicle Speeds in Preemption}

Table 1 lists the test results of the F-test used to determine whether or not the variances of the speeds during preemption and normal operations were equal. The "same," "opposite," and "crossing" in the table indicate the relative direction of the general traffic versus an emergency vehicle. For each segment, both speed during preemption and speed during normal conditions were derived, making their sample size equal $\left(n_{1}=n_{2}\right)$. It can be seen from the table that the variances of average traffic speeds during preemption operations were greater than during normal operations. This result implies that the traffic in preemption may pose a higher safety hazard. 
The t-test was designed to determine whether the speed averages during preemption and normal operations were equal. The bottom part of Table 1 lists the results. Clearly, the general traffic operating in the same direction as emergency vehicles or on crossing streets was slowed down. However, traffic operating in the opposite direction was not slowed by emergency preemption.

\section{Regression Analysis of Vehicle Speeds During Preemptions}

The impact of geometric design elements, traffic characteristics and roadway characteristics on general traffic speed is well documented (for example: Yagar and Aerde 1983, Fitzpatrick et al. 2005, and Garber and Gadiraju 1989).

Table 1: Results of Hypothesis Tests for Speeds

\begin{tabular}{|c|c|c|c|c|c|c|}
\hline \multicolumn{7}{|c|}{ F-Tests } \\
\hline \multirow{2}{*}{$\begin{array}{l}\text { Sample Size } \\
\qquad\left(n_{1}=n_{2}\right)\end{array}$} & \multicolumn{2}{|c|}{ Calculated Variances } & \multirow{2}{*}{$\begin{array}{l}\text { Direction of traffic } \\
\text { with respect to } \\
\text { emergency vehicle }\end{array}$} & \multirow{2}{*}{ F-Value } & \multirow{2}{*}{$\begin{array}{l}\text { F Critical } \\
\text { Value }\end{array}$} & \multirow{2}{*}{ Finding } \\
\hline & Normal & Impacted & & & & \\
\hline 354 & 33.65 & 95.88 & Same & 0.3509 & 1.19 & $\begin{array}{l}\text { Variance } \\
\text { increased }\end{array}$ \\
\hline 419 & 35.70 & 77.32 & Opposite & 0.4616 & 1.17 & $\begin{array}{l}\text { Variance } \\
\text { increased }\end{array}$ \\
\hline 205 & 43.30 & 62.13 & Crossing & 0.6965 & 1.26 & $\begin{array}{l}\text { Variance } \\
\text { increased }\end{array}$ \\
\hline \multicolumn{7}{|c|}{ t-Tests } \\
\hline \multirow{2}{*}{$\begin{array}{l}\text { Sample Size } \\
\qquad\left(n_{1}=n_{2}\right)\end{array}$} & \multicolumn{2}{|c|}{ Calculated Means } & \multirow{2}{*}{$\begin{array}{l}\text { Direction of traffic } \\
\text { with respect to } \\
\text { emergency vehicle }\end{array}$} & \multirow{2}{*}{ t-Value } & \multirow{2}{*}{$\begin{array}{l}\text { t Critical } \\
\text { Value }\end{array}$} & \multirow{2}{*}{ Finding } \\
\hline & Normal & Impacted & & & & \\
\hline 354 & 22.24 & 20.68 & Same & 2.5854 & 1.9640 & $\begin{array}{l}\text { Lower than } \\
\text { Normal }\end{array}$ \\
\hline 419 & 22.26 & 21.82 & Opposite & 0.8292 & 1.9632 & $\begin{array}{c}\text { Not Lower than } \\
\text { Normal }\end{array}$ \\
\hline 205 & 21.87 & 20.13 & Crossing & 2.4270 & 1.9657 & $\begin{array}{l}\text { Lower than } \\
\text { Normal }\end{array}$ \\
\hline
\end{tabular}

Based on availability, the following factors were considered in the regression analysis: (1) duration of signal preemption (seconds), (2) the direction of regular traffic versus the direction of emergency vehicle (opposite direction compared to same direction and crossing), (3) time of the day (peak hour compared to non-peak hour), (4) roadway classification (major street compared to minor street), and (5) transition time (seconds). The response variable was the vehicle speed during signal preemption, "Impacted Speed." Table 2 shows the descriptive statistics for the variables. The duration of signal preemption (labeled as "Pre-duration") was considered because more traffic would be caught in the traffic turbulence caused by emergency preemption if the duration of preemption is very long. Which direction regular traffic was traveling relative to an emergency vehicle may influence the extent of the impact by the emergency vehicle. 
Emergency Vehicle Signal Preemption

Table 2: Descriptive Statistics for Modeling Variables

\begin{tabular}{lccrrrr}
\hline Variable & Type & Unit & Mean & Std. Dev. & Min & Max \\
\hline Impacted Speed & Continuous & Miles Per Hour & 21.0 & 9.1 & 1.9 & 56.2 \\
Preemption Duration & Continuous & Seconds & 25.4 & 13.6 & 6 & 120 \\
Opposite & Binary & & 0.4 & 0.5 & 0 & 1 \\
Peakhour & Binary & & 0.3 & 0.5 & 0 & 1 \\
Major & Binary & & 0.9 & 0.3 & 0 & 1 \\
Transition Time & Continuous & Seconds & 197.6 & 112.4 & 9 & 543 \\
\hline
\end{tabular}

When an emergency vehicle arrives at an intersection, the traffic signal on the crossing streets may either be cut short for green or extended for red. The traffic on the crossing street is expected to be influenced. Such influence may not be significant if the traffic flow on the crossing street was low. In modeling, the dummy variable, labeled as "Opposite," was created to indicate whether regular traffic was in the opposite direction relative to an emergency vehicle. Intuitively, preemptions may have more impact during peak periods. Thus, based on the time period a preemption happened, a dummy variable, labeled as "Peakhour", was considered. The estimated impact of this variable was compared to the base (non-peak condition). Roadway classification was considered in the regression model, because usually the street having more lanes can provide more room for the general traffic to pull over or to allow the emergency vehicle to pass by. Number of lanes may directly be related with roadway functional class. Most of the preemptions in this study happened on major arterials (864 observations) and minor arterials (114 observations). Therefore, only these two road classifications were considered in this model. A dummy variable, "Major," was created to indicate that an emergency vehicle arrived from a major arterial. The estimate of this variable was compared to the base (minor roadway). The variable "Transition" measures the time during which, for coordinated signals, signal phasing and timing transition back to normal operation after a preemption.

Table 3 presents the linear regression results. Consistent with intuition, the preemption duration has a negative association with impacted vehicle speeds. This means that when preemption duration was long, vehicles tended to operate at a lower speed (regardless of where and when it is). The positive coefficient associated with the variable "Opposite" implies that compared to vehicles in the same direction as the emergency vehicle, vehicles in the opposite direction tend to speed up as a result of signal preemption. The traffic in the same direction may be delayed for yielding its rightof-way to the emergency vehicle, while the traffic in the opposite direction may fully take advantage of the extended green time. Note that at most intersections, when the signal facing the emergency vehicle turns green, also those facing the opposing traffic turn green. The negative coefficient for the variable "Peakhour" implies that traffic, regardless of its relative location, moved relatively slower when a preemption happened in peak period than when it happened during off-peak period. This is due to the fact that more traffic is influenced by preemptions in this period than others. The coefficient of the variable "Major" is positive, which reflects the situation that traffic on major roadways move at higher speeds due to availability of room to yield the right-of-way to an emergency vehicle (in the case of vehicles moving in the same direction as the emergency vehicle). Similar to preemption duration, transition time has a negative impact on vehicle speeds.

Variables "Opposite" and "Major" are significant at the 0.01 level. "Peakhour" is significant at the 0.05 level, and "Pre-duration" and "Transition" are significant at the 0.10 level. The $\mathrm{R}^{2}$ value is low, but the purpose of the analysis is hypothesis testing not forecasting. It should be noted that the model is significant according to the F-test (p-value of 0.0001). 
Table 3: Results of Regression Analysis

\begin{tabular}{lcccc|cc}
\hline \multicolumn{1}{c}{ Variables } & Coefficient & Std. Err. & t-value & p-value & \multicolumn{2}{c}{ 90\% Conf. Interval } \\
\hline Pre-duration & -0.040 & 0.021 & -1.88 & 0.060 & -0.0747 & -0.0050 \\
Opposite & 1.660 & 0.583 & 2.85 & 0.005 & 0.7000 & 2.6196 \\
Peakhour & -1.609 & 0.643 & -2.50 & 0.013 & -2.6671 & -0.5502 \\
Major & 2.900 & 0.900 & 3.22 & 0.001 & 1.4189 & 4.3818 \\
Transition & -0.005 & 0.003 & -1.80 & 0.073 & -0.0091 & -0.0004 \\
Constant & 19.514 & 1.073 & 18.18 & 0.000 & 17.7471 & 21.2812 \\
\hline Number of obs $=978$ & & & & & & \\
F ( 5, 972) $=6.28$ & & & & & \\
Prob $>$ F $=0.0001$ & & & & & & \\
R-squared $=0.0316$ & & & & & & \\
Adj R-squared $=0.0263$ &
\end{tabular}

\section{CONCLUSIONS, RECOMMENDATIONS, AND FUTURE STUDY NEEDS}

This study evaluates the impact of emergency vehicle signal preemption on traffic operations in the Las Vegas Urbanized Area. The evaluation of the impacts of preemption on traffic conditions focused on comparison of traffic speeds during preemption operations with those in normal operations. Hypothesis tests were performed on the variance of traffic speeds during preemption versus those in normal operations. The results showed that the variance of traffic speed during preemptions was significantly larger than during normal operations, which verifies the speed turbulence caused by an emergency vehicle on regular traffic. Such variance may have a significant impact on traffic safety on the road (Varhelyi et al. 2003, and Garber and Gadiraju 1989). A hypothesis test was also conducted on the means of traffic speeds during preemption operations and those during normal operations. The results indicated that the emergency vehicle slowed down the traffic only in the same direction and on crossing streets.

In addition to the hypothesis tests, the influence of roadway and traffic characteristics on speed of traffic was analyzed using a regression model. The results show that duration of preemption has a negative association with speeds. Similarly, preemption during the peak-hour has a significant negative impact on traffic speeds compared to preemption during non-peak time. The location of traffic relative to the emergency vehicle was also evaluated. It was found that traffic running in the opposite direction of the emergency vehicle tends to have higher speed as a result of signal preemption when compared to those running in the same direction as the emergency vehicle. Also, the results showed that traffic running on major roadways tends to have higher speed. This may be due to availability of room for maneuvering compared to minor roadways. Similar to preemption duration, transition time has a negative impact on vehicle speeds.

While it is difficult to control the time of the day (peak or non-peak) during which signal preemption is needed, it is possible to optimize (minimize) the preemption duration, as well as transition time. The results of this study suggest that traffic in the opposite direction tends to have higher speed as a result of signal preemption. This suggests that a study on safety implications of higher speeds of traffic in the opposite direction is needed. Also, in this study the locations of traffic relative to an emergency vehicle were classified into (1) the same direction as the emergency vehicle, (2) the opposite direction, and (3) on crossing streets. It may also be worthwhile to further break down and classify the location of the same direction traffic to "leading an emergency vehicle" and "trailing an emergency vehicle." By knowing these locations, the impact of emergency vehicles on the speed of traffic in the same direction of an emergency vehicle can be more precisely determined. Due to the limitation of the data available to this study, such identification of the location of general traffic was impossible. It is recommended that the GPS data be collected in a shorter distance 
Emergency Vehicle Signal Preemption

interval, which would make such identification possible. In addition, the impacts of presence of raised median as well as the number of lanes need to be studied.

\section{References}

Bullock, D., J. Morales, and B. Sanderson Jr. "Impact of Signal Preemption on the Operation of the Virginia Route 7 Corridor." Submitted to the 1999 ITS America Annual meeting for presentation and publication, 1999.

Casturi, R., W. Lin, and J. Collura. "A Macroscopic Model for Evaluating the Impact of Emergency Vehicle Signal Preemption on Traffic." Paper presented at the $79^{\text {th }}$ Annual Transportation Research Board Meeting, Washington, D.C., 2000.

Fitzpatrick, K., S. P. Miaou, M. Brewer, P. Carlson, and M. D. Wooldridge. "Exploration of the Relationships Between Operating Speed and Roadway Features on Tangent Sections." Journal of Transportation Engineering 131 (4), (2005): 261-269.

Garber, N. J. and R. Gadiraju. "Factors Affecting Speed Variance and Its Influence on Accidents." Transportation Research Record 1213, (1989): 64-71.

Gifford, J., D. Pelletiere, and J. Collura. "Stakeholder Requirements for Traffic Signal Preemption and Priority in the Washington, D.C., Region.” Transportation Research Record 1748, (2001): 1-7.

Kaseko, M. and H. Teng. "Evaluation of Operations of Signal Preemption for Emergency Vehicles to Enhance Traffic Flow.” Draft Project Proposal, 2005.

Nelson, E. J. and D. Bullock. "Impact of Emergency Vehicle Preemption on Signalized Corridor Operation: An Evaluation.” Transportation Research Record 1727, (2000): 1-11.

Várhelyi, A., M. Hjälmdahl, C. Hydén, and M. Draskóczy. "Effects of an Active Accelerator Pedal on Driver Behaviour and Traffic Safety after Long-term Use in Urban Areas." Accident Analysis \& Prevention 36 (5), (2004): 729-737.

Yagar, S. and M. V. Aerde. "Geometric and Environmental Effects On Speeds of 2-Lane Highways." Transportation Research Part A 17A, (1983): 315-325.

Yun, I., M. Best, and B. Park. "Evaluation of Emergency Vehicle Preemption Strategies on a Coordinated Actuated Signal System Using Hardware-in-the-loop Simulation." Paper presented at the $86^{\text {th }}$ Annual Transportation Research Board Meeting, Washington, D.C., 2007. 
Hualiang (Harry) Teng is a faculty member in the Department of Civil and Environmental Engineering at the University of Nevada, Las Vegas. He received his Ph.D. from Purdue University. His research interests are in the areas of Intelligent Transportation Systems, transportation planning, demand modeling, air quality analysis, and freight transportation systems.

Valerian Kwigizile is an assistant professor of civil engineering at the West Virginia University Institute of Technology, Montgomery, WV. He earned his Ph.D. from the University of Nevada, Las Vegas, and a masters degree from the Florida State University. He also holds a B.S. civil engineering degree from the University of Dar-Es-Salaam, Tanzania. His research interests include transportation modeling and planning, traffic safety, simulation and operations, application of intelligent transportation systems, air quality analysis, and GIS application in civil engineering. He is a member of the Institute of Transportation Engineers and an associate member of the American Society of Civil Engineers.

Gang Xie has a master's degree in civil engineering from University of Nevada, Las Vegas. He also obtained a master's degree and bachelor's degree in transportation from Northern Jiaotong University in Beijing, China. He specializes in data processing and analysis, GIS applications in transportation, and developing transportation related software. Xie is currently working as project engineer at the Regional Transportation Commission (RTC) of Southern Nevada, Freeway and Arterial System of Transportation (FAST). His major responsibility is to design and develop performance measurement systems for freeways and arterials in the Las Vegas metropolitan area.

Mohamed Kaseko is an associate professor of civil engineering at the University of Nevada, Las Vegas. His field of specialty is transportation engineering. He has several years of experience in research and teaching in transportation planning, transportation and traffic operations, traffic safety and Intelligent Transportation Systems. He has authored and co-authored several papers and conference presentations in these areas. Kaseko received his Ph.D. in civil engineering from the University of California at Irvine, M.S. from Cornell University, and B.Sc. (engineering) from the University of Dar es Salaam, Tanzania.

Presently, A. Reed Gibby has part-time research appointments at both the Las Vegas and Reno campuses of the University of Nevada as a senior transportation research engineer. After graduating with a B.S. degree from Brigham Young University in 1967 as a civil engineer, he spent 17 years in transportation at the state, regional and local levels in Washington, Oregon, California and Alaska. This experience included: highway design; traffic control, operations and maintenance; and multimodal transportation planning and programming. In 1984, he joined the civil engineering faculty at California State University, Chico, where he taught mostly transportation courses and conducted transportation research. During the 19 years in Chico, he was involved with more than two dozen research projects. After retiring from Chico in 2003 and being granted emeritus professor status, he joined Salaegui Engineers in Sparks, NV, and then joined the Nevada DOT Research Division in 2004. He finished his career with Nevada DOT in January, 2010, as chief operations analysis engineer. He earned his M.S. degree from UC Berkeley and Ph.D. from University of California, Davis, in transportation Engineering. He is a professional engineering in Washington and Nevada, and has been active in Institute of Transportation Engineers, Transportation Research Board and American Society of Civil Engineers. He has also been an expert witness for traffic accidents in Washington, California, Alaska and Utah. 